\title{
Asymmetric kinase dimer formation is crucial for the activation of oncogenic EGFRvill but not for ERBB3 phosphorylation
}

\author{
Rama Krishna Kancha, Nikolas von Bubnoff and Justus Duyster ${ }^{*}$
}

\begin{abstract}
Background: Formation of asymmetric kinase dimers is required for wt-EGFR activation upon ligand stimulation. The role of receptor dimerization in oncogenic EGFRvIll mutant activation is not completely understood and the molecular details of EGFRvIll interactions within homo-dimers and hetero-dimers are not elucidated yet.

Findings: By employing mutations that disrupt the asymmetric kinase dimer interface in EGFRvIll, we demonstrate that the mechanism of oncogenic EGFRvIll mutant activation is similar to that of the full-length wild-type EGFR. Surprisingly, the monomeric EGFRvIll lacks autophosphorylation and the formation of asymmetric kinase dimers is indispensable for oncogenic kinase activation. In addition, we show that ERBB3 can act as an activator of EGFRvIll by forming asymmetric kinase dimer in a ligand-independent manner. Interestingly, we found that the formation of asymmetric kinase dimer is dispensable for ERBB3 phosphorylation by the activated EGFR kinase as well as the ERBB2 kinase thus revealing a novel model for receptor function.
\end{abstract}

Conclusions: Lateral signaling is a novel mechanism of signal propagation via ERBB3 upon activation by EGFR/ERBB2 kinase even in the absence of their ability to form asymmetric kinase dimers.

Keywords: EGFRvIII, Asymmetric dimer, ERBB3, ERBB2, Lateral signaling

\section{Findings}

\section{EGFRvIll monomers lack kinase activity}

Previous studies reported that EGFRvIII dimers cannot be detected in cells suggesting that kinase-active monomers of EGFRvIII execute mitogenic signal transduction [1-4]. This hypothesis is in accordance with the fact that EGFRvIII lacks the ligand-binding domain, which is critical for receptor dimerization. On the other hand subsequent reports were able to detect EGFRvIII dimers and could demonstrate that their activity is comparable to that of the ligand-stimulated wild-type EGFR $[5,6]$. Thus, the role of EGFRvIII dimerization for kinase activation is controversial and not fully elucidated. Recently, Zhang et al. using an elegant approach were able to demonstrate that the formation of asymmetric dimers of the kinase domain are absolutely essential for wt-EGFR activation upon ligand stimulation [7]. To test if asymmetric

\footnotetext{
* Correspondence: direktion-med1@uniklinik-freiburg.de Department Medicine I, University Medical Center Freiburg, Freiburg, Germany
}

(c) 2013 Kancha et al.; licensee BioMed Central Ltd. This is an Open Access article distributed under the terms of the Creative Commons Attribution License (http://creativecommons.org/licenses/by/2.0), which permits unrestricted use, distribution, and reproduction in any medium, provided the original work is properly cited. dimer formation is important for kinase activity we introduced point mutations (Additional file 1) in either the N-lobe or C-lobe of EGFRvIII and EGFRvIII-D837N (kinase-dead) (Figure 1A-B, Additional file 2: Figure S1A). We used cells that lack endogenous EGFR expression $[8,9]$ (Figure 1A, lane 1). Disruption of the asymmetric kinase dimer interface by either N-lobe I706Q mutation (Figure 1A, lane 4) or C-lobe V948R mutation (Figure 1A, lane 5) indeed abrogated EGFRvIII kinase activity compared to the unmutated control (Figure 1A, lane 2). This data indicate that an intact asymmetric kinase dimer interface is essential for EGFRvIII kinase activation and was an unexpected finding given the previous observation that the receptor is not able to efficiently form stable dimers (Figure 1B, Additional file 2: Figure S1A) [1].

To further demonstrate the importance of asymmetric dimer formation for kinase activity, cells were transfected with a combination of mutants wherein the kinase activity of a C-lobe mutant (V948R) is rescued in trans by the kinase-dead (D837N) EGFRvIII either alone (Figure 1A-B, lane 7) or in combination with the N-lobe 
A

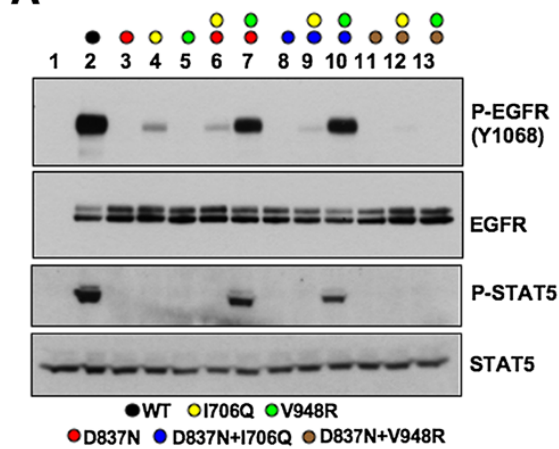

B

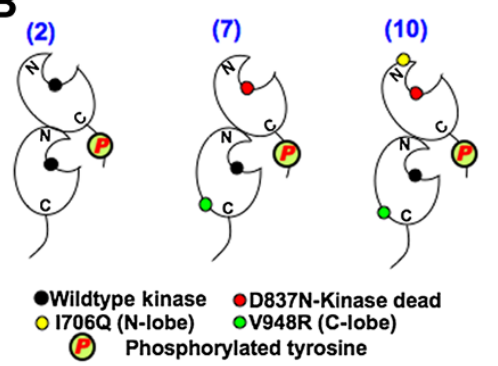

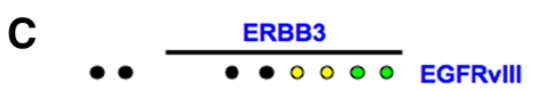

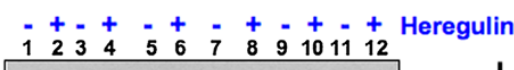
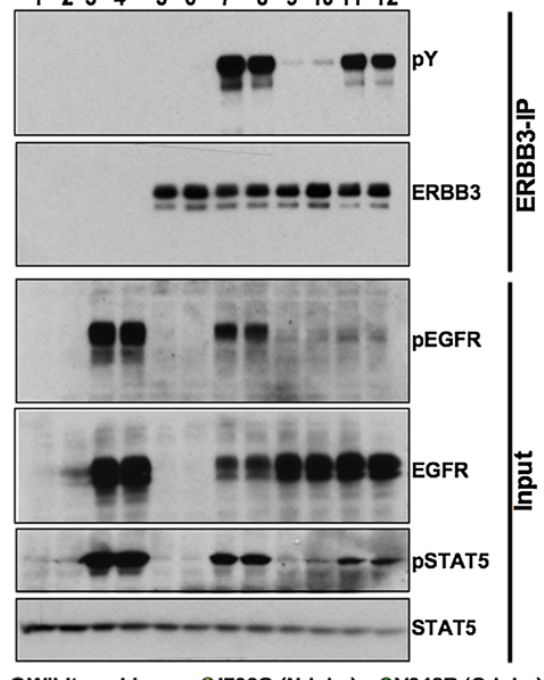

-Wildtype kinase OI706Q (N-lobe) OV948R (C-lobe)

D

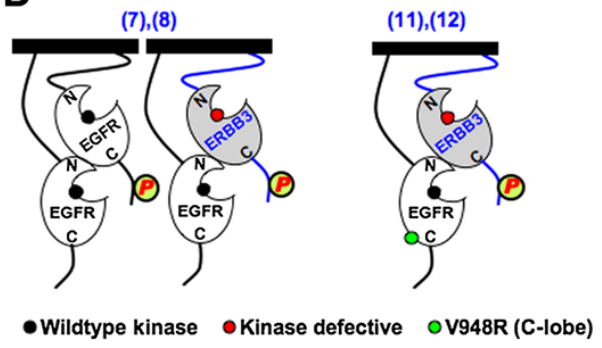

Figure 1 Asymmetric kinase dimer formation is involved in EGFRvIll activation and ERBB3 phosphorylation. (A) EGFRvIII point mutations were transfected into HEK293 cells either alone or in combinations as indicated by colored circles (lanes 2 to 13). Untransfected cells were taken as negative control (lane 1). Cell lysis was performed 36 hours after transfection followed by SDS-PAGE. Immunoblotting was performed with anti-p-EGFR (Y1068) (upper panel), anti-EGFR (second panel), anti-p-STAT5 (third panel) and anti-STAT5 (lower panel) antibodies. (B) Schematic representation of the most important mechanisms of wild-type and mutant EGFRvlll kinase activation corresponding to the experiments shown in Figure 1A in lanes 2, 7 and 10. Kinases were labeled black (intact active centre) or red (kinase dead) and yellow (N-lobe mutant) or green (C-lobe mutant) circles. (C) Wild-type or mutated EGFRvill kinase (1706Q or V948R) was co-transfected with wild-type ERBB3 in HEK293 cells. After 36 hours, serum starvation for 12 hours was performed. Cells then were stimulated or not with $50 \mathrm{ng} / \mathrm{ml}$ heregulin for 5 minutes before cell lysis was performed. Immunoprecipitates with anti ERBB3 (upper 2 panels) or whole cell lysates (lower four panels) were resolved by SDS-PAGE and western blotting was performed with the antibodies indicated. (D) Schematic representation of key kinase domain interactions in receptor homo- and hetero-dimers corresponding to the experiments shown in Figure $1 \mathrm{C}$ in lanes 7, 8, 11 and 12.

mutant (Figure 1A-B, lane 10). In contrast, the activity of C-lobe (V948R) mutant could not be rescued by a kinase-dead C-lobe mutant (D837N + V948R) due to the disruption of the asymmetric kinase dimer interface (Figure 1A-B, lane 13). Additional EGFRvIII mutants with disrupted asymmetric kinase dimer interface both in wild-type and D837N background were taken as controls (lanes 6, 8, 9, 11 and 12) to demonstrate the absence of cis-autophosphorylation (Figure 1A-B, Additional file 2: Figure S1A). Together these data argue for an important role of asymmetric dimer formation also for EGFRvIII kinase activation. Furthermore, it shows for the first time that the extra-cellular in-frame deletion of the EGFRvIII receptor does not result in an activated monomer as previously anticipated. A recent study reported the importance of Cys307 (wild-type EGFR numbering; Cys16 in mature EGFRvIII mutant) in EGFRvIII receptor dimerization [10]. We thus cloned the C16S mutant into the EGFRvIII/ D837N backbone and tested for its ability to activate the C-lobe mutated EGFRvIII. As expected, the EGFRvIII/ $\mathrm{C} 16 \mathrm{~S}+\mathrm{D} 837 \mathrm{~N}$ mutant was not able to activate the EGFRvIII/V948R mutant indicating that the receptor dimerization is indispensable for EGFRvIII activity (Additional file 2: Figure S1B). 
ERBB3 is an activator of EGFRvIII in an asymmetric kinase hetero-dimer

Recently, it was shown that ERBB3 could act as an activator for the wt-EGFR kinase [11]. However, it is not known whether oncogenic EGFRvIII is able to form activating dimers with ERBB3. To test for potential ERBB3/ EGFRvIII interactions, we expressed both constructs in HEK293 cells, which lack ERBB receptor expression [8,12] (Figure 1C: lanes 1-2, Additional file 3: Figure S2A). ERBB3 lacks intrinsic kinase activity [11] and when expressed alone didn't cause receptor phosphorylation even in the presence of it's ligand heregulin (Figure $1 \mathrm{C}$ : lanes 5-6, Additional file 3: Figure S2A). However, expression of ERBB3 together with EGFRvIII mutant resulted in ERBB3 phosporylation indicating that ERBB3 can act as a substrate for EGFRvIII kinase by forming heterodimers (Figure 1C-D: lanes 7-8). Interestingly, EGFRvIII-I706Q (N-lobe) mutant that disrupts asymmetric kinase dimer formation didn't result in ERBB3 phosphorylation indicating that ERBB3 acts as an activator of EGFRvIII kinase (Figure 1C: lanes 9-10, Additional file 3: Figure S2A). Moreover, ERBB3 rescued the kinase activity of EGFRvIIIV948R (C-lobe) mutant thus demonstrating that the asymmetric kinase dimer interface is similar for both the EGFRvIII homodimers and EGFRvIII-ERBB3 heterodimers (Figure 1C-D: lanes 11-12). Receptor phosphorylation also correlated with the phosphorylation of downstream targets such as STAT5 indicating that the asymmetric kinase dimers are indeed functional. The phenomenon of EGFR kinase activation by asymmetric kinase dimerization thus seems to be highly conserved among different members of the EGFR family and different types of activating mutations found in human cancers $[7,13]$. Since kinase-inactive EGFRvIII is still able to be an activator for a partner receptor, inhibitor-bound-EGFRvIII may still activate other receptors of the EGFR family (ERBB2 or ERBB4). In the setting of EGFR tyrosine kinase inhibitor (TKI) treatment this may lead to altered signal transduction and secondary drug resistance. Thus, the expression of ERBB2, ERBB3 and ERBB4 in EGFR driven cancer may be important to predict the outcome of TKI treatment.

\section{Asymmetric kinase dimerization is dispensible for ERBB3 phosphorylation by activated EGFR and ERBB2 kinases}

The dynamic role of monomers within an asymmetric dimer is not completely understood. It has been postulated that reciprocal asymmetric dimer formation, i.e. the acceptor kinase becomes the activator and vice versa leads to the full activation of both monomers [14]. It was also hypothesized that the activation by asymmetric kinase dimerization may happen in higher-order oligomers [14]. To address this we employed the EGFRvIIIERBB3 heterodimer as a model wherein kinase activation can be viewed separately from substrate phosphorylation due to the differences in both size and the epitope. A series of EGFRvIII and ERBB3 mutants were created for this purpose. Consistent with the data shown in Figure 1C, EGFRvIII was able to phosphorylate ERBB3 in a ligand independent manner (Figure 2A-B: lanes 3,4). ERBB3-V945R was previously shown to disrupt asymmetric kinase dimer formation [11] and as expected it failed to activate $\mathrm{C}$-lobe (acceptor) mutant EGFRvIII (Figure 2A: lanes 5, 6, Additional file 3: Figure S2B). Surprisingly, when expressed together with an "activated EGFRvIII receptor homodimer unit”, ERBB3V945R was phosphorylated (Figure 2A-B: lanes 7,8). This phenomenon was not observed when the "kinase-defective EGFRvIII activation unit" was used (Figure 2A: lanes 9,10, Additional file 3: Figure S2B) indicating that EGFRvIII is indeed the kinase. This finding is unexpected since ERBB3-V945R is defective in forming asymmetric kinase dimer with either of the EGFRvIII monomers used (EGFRvIII-D837N/I706Q and EGFRvIII-V948R). Thus, the lack of ability to act as an activator-kinase did not disqualify C-lobe (V945R) mutant ERBB3 to act as a substrate for EGFRvIII kinase. Symmetric kinase dimers were reported in EGFR crystals even though they have no role in kinase activation [7]. To test their role we introduced mutations (R962E and K970E) that disrupt the symmetric kinase interface of the activated (acceptor) kinase and expressed it together with ERBB3. Disruption of symmetric kinase dimer interface didn't abrogate the ability of activated EGFRvIII kinase to phosphorylate ERBB3 (Figure 2C). Interestingly, a slight decrease in ERBB3 phosphorylation was observed upon heregulin stimulation (Figure 2C). It was previously shown that heregulin stimulation results in the destabilization of ERBB3 oligomers but not of receptor dimers [15]. Thus, the observed reduction of ERBB3 phosphorylation upon heregulin stimulation in this particular setting might be due to the disruption of higher-order oligmers involving ERBB3 receptors.

To test if similar mechanisms are involved in ERBB3 phosphorylation by the full-length receptor, we expressed both wild-type and C-lobe mutant ERBB3 together with the wild-type EGFR. Interestingly, full length, wild-type EGFR phosphorylated both wild-type as well as the C-lobe mutant ERBB3 upon EGF stimulation but not upon heregulin stimulation (Figure 2D). EGFR-L858R, a constitutively active mutant that was reported in NSCLC patients, also phosphorylated both wt and C-lobe mutant ERBB3 in a ligand-independent manner (Figure 2E). These results imply that it is not necessary for ERBB3 to be part of an asymmetric kinase dimer to be activated by EGFR kinase. The phosphorylation of ERBB3 by EGFR kinase despite the inability to form neither asymmetric nor symmetric dimers of kinase domain indicates that the 
A
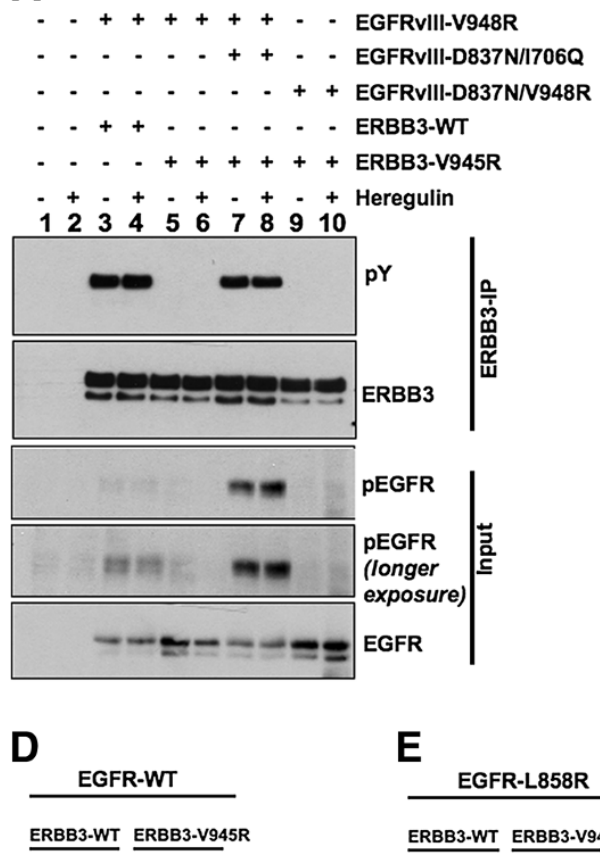

-++-+ Heregulin

-+-+ - EGF
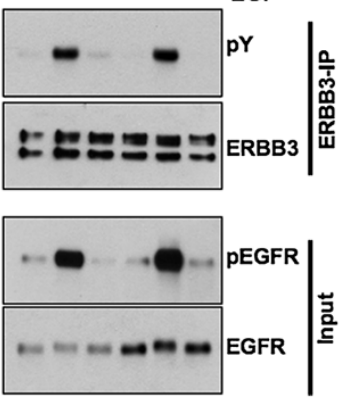

B

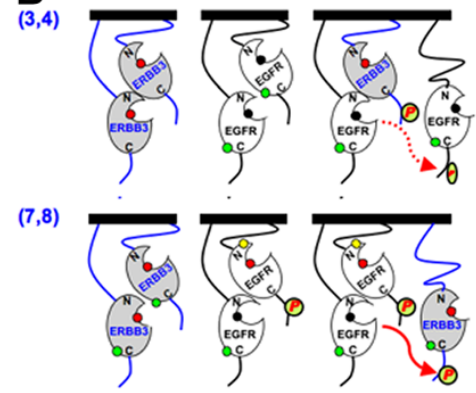

C ERBB3-V945R
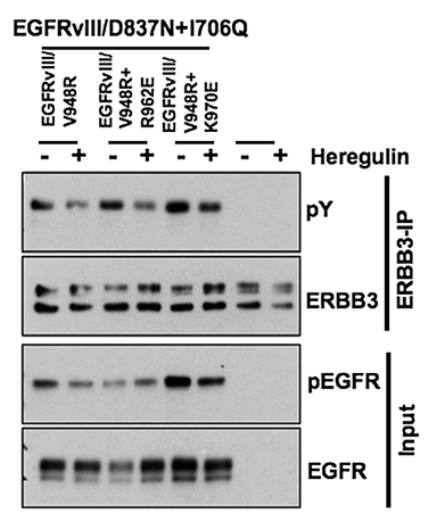

=-503 ERBB3

E EGFR-L858R

ERBB3-WT ERBB3-V945R

.-++-+ Heregulin
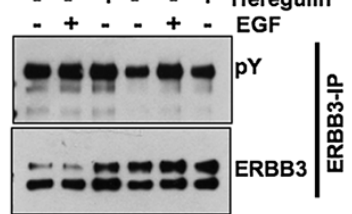

$\because \because m=n$ EGFR 芫

\section{물}

Figure 2 Asymmetric kinase dimer formation is dispensable for ERBB3 activation by EGFR kinase. (A) Indicated EGFRVIII and ERBB3 constructs were transfected into HEK293 cells. After 36 hours, stimulation with $50 \mathrm{ng} / \mathrm{ml}$ of heregulin for 5 minutes was performed. Cells were then lysed and subjected to immunoprecipitation using anti-ERBB3 antibody. Immunoblotting was performed on ERBB3 immunoprecipitates with indicated antibodies (top 2 panels). Whole cell lysates (input) were subjected to western blotting with indicated antibodies (lower 3 panels). (B) Schematic representation of receptor interactions corresponding to the experiments shown in Figure 2A (lanes 3, 4, 7 and 8). (C) Indicated EGFR and ERBB3 mutants were transfected into HEK293 cells followed by serum starvation and heregulin (50 ng/ml) stimulation for 5 minutes. Immunoblotting on ERBB3 immunoprecipitates was performed with indicated antibodies (upper 2 panels). Immunoblotting of whole cell lysates (input) was performed with indicated antibodies (lower 2 panels). (D) Wild-type EGFR was co-transfected with either wild-type or C-lobe mutant (V945R) ERBB3 into HEK293 cells. After 36 hours, cells were serum starved for 12 hours followed by stimulation with EGF (25 ng/ml) or heregulin $(50 \mathrm{ng} / \mathrm{ml})$ for 5 minutes. Cells were then lysed and subjected to ERBB3 immunoprecipitation. SDS-PAGE and immunoblotting on ERBB3 immunoprecipitates was performed with indicated antibodies (upper 2 panels). Western blotting on whole cell lysates (input) was performed with indicated antibodies (lower 2 panels). (E) EGFR-L858R was co-transfected with either wild-type or C-lobe mutant (V945R) ERBB3 into HEK293 cells. After 36 hours, cells were serum starved for 12 hours followed by stimulation with EGF ( $25 \mathrm{ng} / \mathrm{ml})$ or heregulin $(50 \mathrm{ng} / \mathrm{ml})$ for 5 minutes. Cells were then lysed and subjected to ERBB3 immunoprecipitation. SDS-PAGE and immunoblotting on ERBB3 immunoprecipitates was performed with indicated antibodies (upper 2 panels). Western blotting on whole cell lysates (input) was performed with anti-EGFR antibody.

dimerization or oligomerization at receptor level brings the acceptor kinase close enough to phosphoryate it's substrate (Figure 3A). Moreover, ERBB2 phosphorylated both the wild-type and C-lobe mutant ERBB3 (Figure 3B) indicating that the observed mechanism is conserved among different members of the ERBB family.

\section{Activated ERBB3 potentiates the transforming ability of} EGFR and ERBB2

To test the functional role of kinase activation within different ERBB receptor combinations, we employed a competition assay using $\mathrm{Ba} / \mathrm{F} 3$ cells. $\mathrm{Ba} / \mathrm{F} 3$ cells lack ERBB receptor expression and were previously used to 

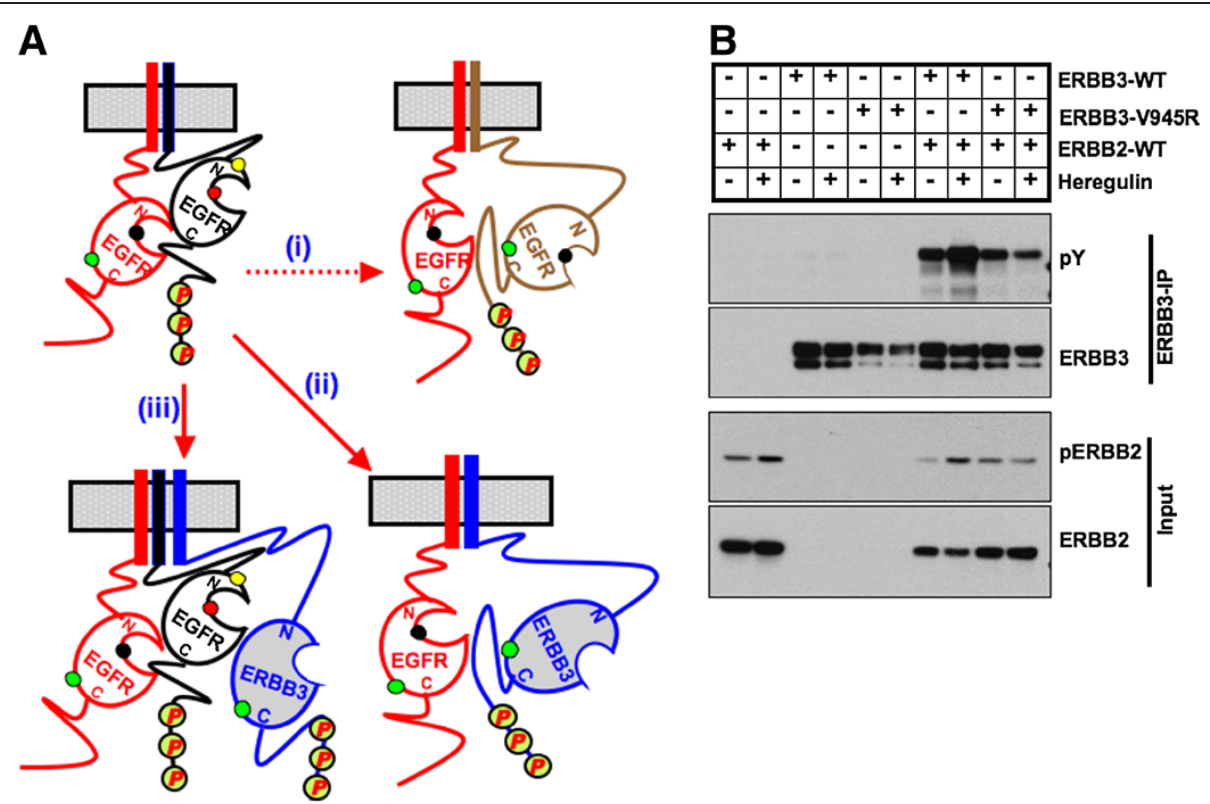

Figure 3 Activated EGFR/ERBB2 kinase phosphorylates ERBB3 independent of asymmetric kinase dimer formation. (A) Schematic representation of two hypothetic mechanisms potentially involved in activator function-defective EGFR/ERBB3 phosphorylation by activated EGFR: The activated EGFR dissociates from the activator kinase to phosphorylate another inactive (activator function-defective) EGFR (i) or ERBB3 (ii) receptor. Alternatively, activated EGFR may form higher-order oligomer or hetero-tetramer complexes to cause phosphorylation of activator function-defective ERBB receptors (iii). (B) HEK293 cells were transfected with wild-type ERBB2 either alone or together with WT or mutant ERBB3 (V945R). After 36 hours, cells were serum starved for 12 hours followed by stimulation with $50 \mathrm{ng} / \mathrm{ml}$ heregulin for 5 minutes as indicated. Cells were then lysed in TMNSV buffer [16]. Cells lysates were subjected to immunoprecipitation using rabbit anti-ERBB3 antibody and immunoblotting was performed using mouse anti-phospho-tyrosine and mouse anti-ERBB3 antibodies (upper 2 panels). Western blotting on whole cell lysates (input) was performed with anti-pERBB2 and anti-ERBB2 antibodies (lower 2 panels).

test the transformation potential of ERBB receptors $[8,9,12]$. Ba/F3 cells require IL-3 for survival and introduction of oncogenes into these cells confer cytokineindependence on these cells $[8,9,12]$. Thus, we employed $\mathrm{Ba} / \mathrm{F} 3$ cells to test the physiological role of ERBB3 phosphorylation by ERBB receptors in different combinations. Stable cell lines expressing either wild-type or C-lobe mutant ERBB3 (V945R) were established for this purpose (Figure 4A). Both wild-type and C-lobe mutant ERBBs (EGFR, EGFRvIII and ERBB2) were transduced in to either parental $\mathrm{Ba} / \mathrm{F} 3$ cells (Figure 4B-E: panel i) or Ba/F3 cells that stably express ERBB3 receptors (Figure 4B-E: panels ii and iii). Since EGFR, EGFRvIII and ERBB2 were expressed from bi-cistronic vectors that co-express either GFP or YFP, the signaling strength of receptor combinations was measured as the rate of outgrowth of transduced cells over untransduced cells $[9,12]$. EGFRvIII transformed $\mathrm{Ba} / \mathrm{F} 3$ cells to cytokine-independence as reported previously (Figure 4B: i) $[8,9]$. The transformation of $\mathrm{Ba} / \mathrm{F} 3$ cells by EGFRvIII is potentiated by the co-expression of ERBB3 receptor indicating an important role of ERBB3 phosphorylation (Figure 4B: ii, iii). As expected, the C-lobe mutant EGFRvIII-V948R didn't transform Ba/ F3 cells (Figure 4C: i). However, expression of ERBB3 receptor conferred IL3-independence on $\mathrm{Ba} / \mathrm{F} 3$ cells that stably express EGFRvIII-V948R indicating the functional role of asymmetric kinase dimer formation (Figure 4C: ii). EGFRvIII-V948R however failed to transform $\mathrm{Ba} / \mathrm{F3}$ cells that expressed ERBB3-V945R even after heregulin stimulation indicating the importance of asymmetric kinase dimer interface in the activation of receptor (Figure 4C: iii). Moreover, transformation of $\mathrm{Ba} / \mathrm{F} 3$ cells to IL-3 independence by full-length wild-type EGFR was also potentiated by ERBB3 upon heregulin stimulation (Figure 4D: i, ii). Even though similar patterns were seen with cells expressing ERBB3-V945R, the transformation ability was relatively weak compared to that of cells expressing wild-type ERBB3 (Figure 4D: ii, iii). Similar observations were made with the combination of ERBB2 and ERBB3 receptors (Figure 4E: i - iii) indicating that the signaling strength resulting from the lateral signaling is weaker compared to that of the 'activating asymmetric kinase dimer' unit.

\section{Conclusions}

Formation of asymmetric kinase dimer is essential for both the constitutive activation of oncogenic EGFR as well as the ligand-stimulated wild-type EGFR. However, phosphorylation of ERBB3 by the activated EGFR or ERBB2 kinase may occur in higher order oligomers in 

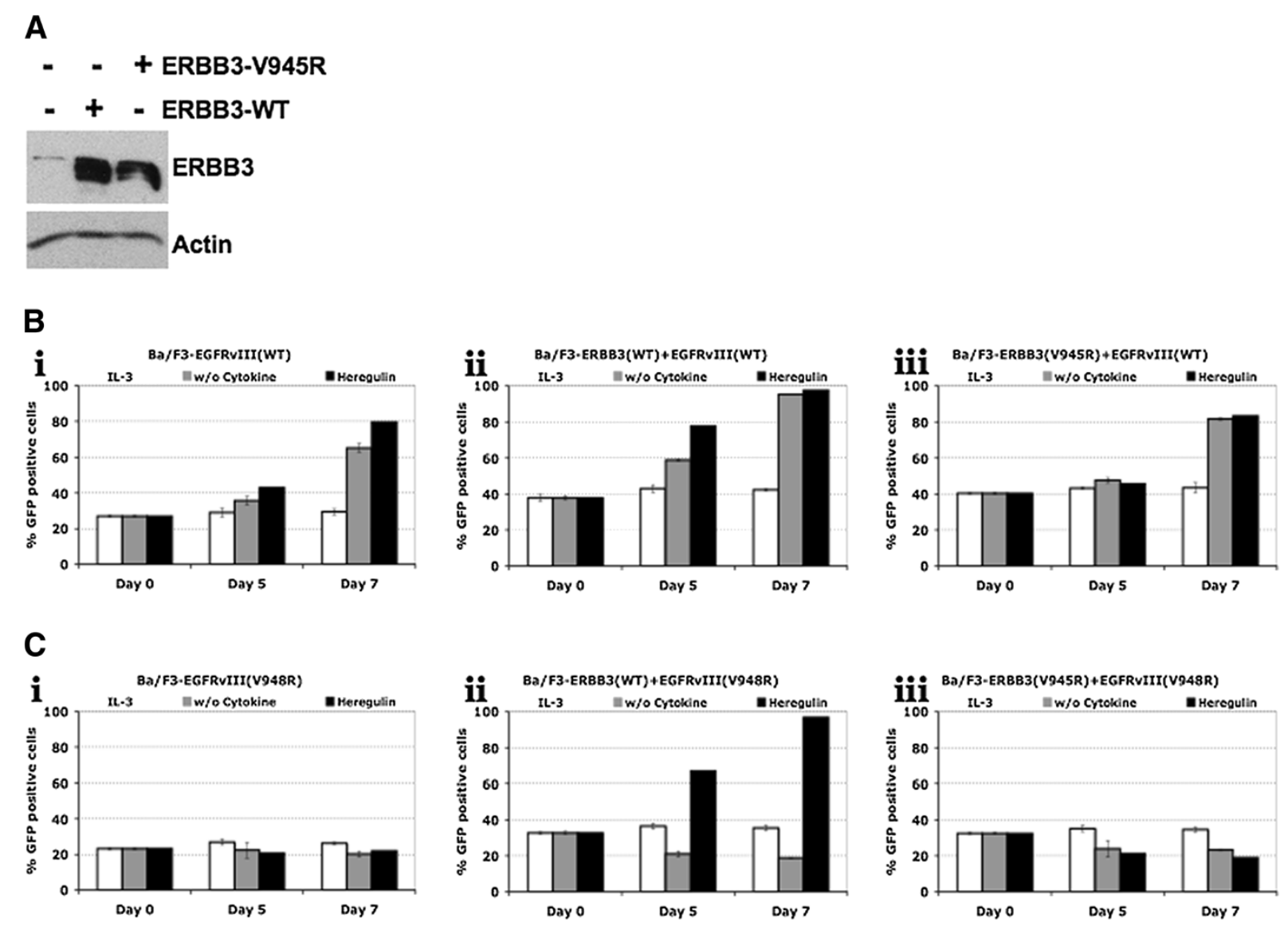

D
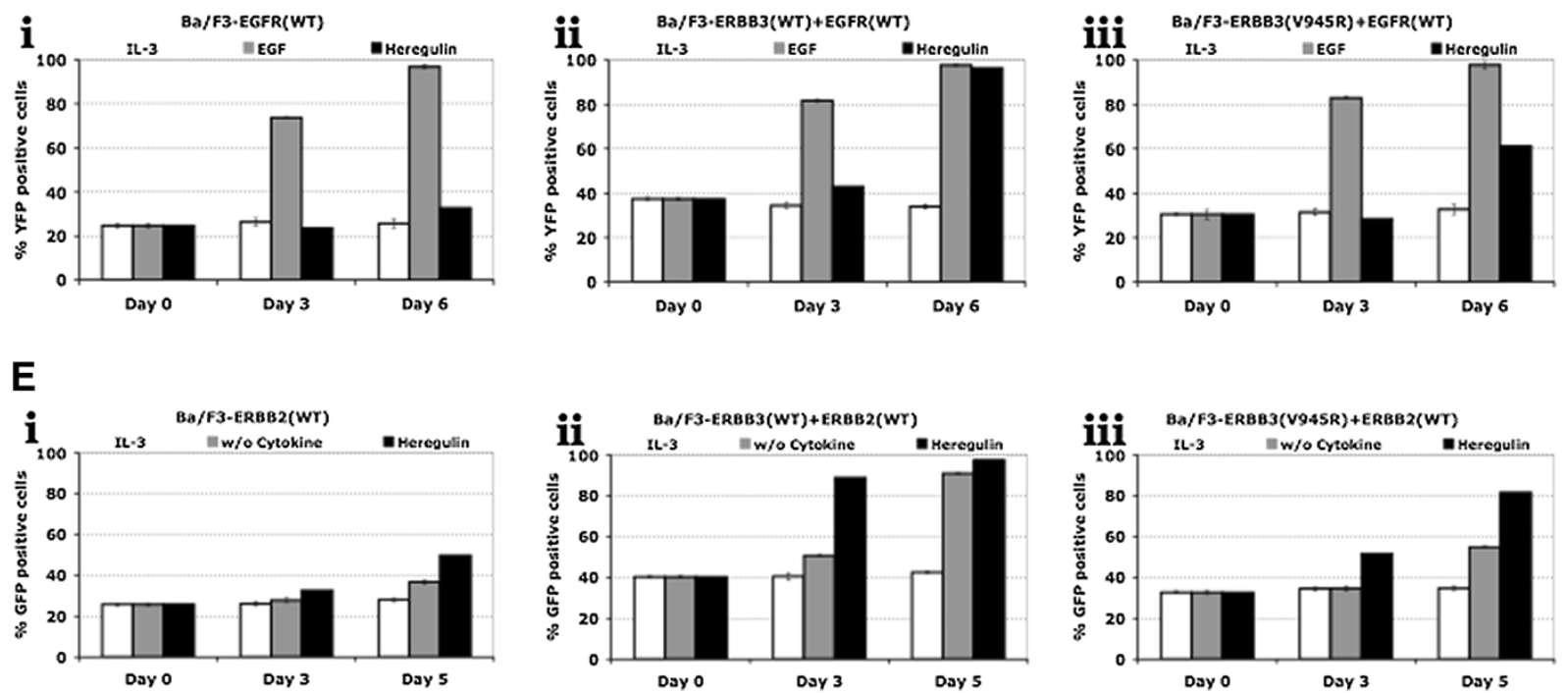

Figure 4 Functional analysis of ERBB heterodimers. (A) Ba/F3 cells stably expressing indicated ERBB3 receptors were lysed and western blotting was performed with anti-ERBB3 and anti-beta-actin antibodies. Untransfected Ba/F3 cells were taken as a negative control. (B-E) Ba/F3 cell lines described above with or without stable expression of ERBB3 constructs were additionally infected with MSCV-GFP-EGFRvIll (B: i: control, ii: ERBB3-WT, iii: ERBB3-V945R), MSCV-GFP-EGFRvIIIN948R (C: i: control, ii: ERBB3-WT, iii: ERBB3-V945R), MSCV-YFP-EGFR (D: i: control, ii: ERBB3-WT, iii: ERBB3-V945R) or MSCV-GFP-ERBB2 (E: i: control, ii: ERBB3-WT, iii: ERBB3-V945R). Cells were then cultured with or without the indicated cytokines (IL-3, EGF $(25 \mathrm{ng} / \mathrm{ml})$ or heregulin $(50 \mathrm{ng} / \mathrm{ml})$. The Percentage of GFP-positive (EGFRvIll or ERBB2) or YFP (EGFR-WT)-positive cells was measured by FACS analysis at the indicated time points. 
the absence of asymmetric kinase dimer formation. Thus, asymmetric kinase dimer formation plays a differential role in EGFR receptor activation and ERBB3 phosphorylation. Recent studies have implicated the role of ERBB3 as a critical heterodimeric partner for both EGFR and ERBB2 in drug resistance [17]. Since the formation of receptor complexes is important for their activity, the use of antibodies that target ERBB receptors either alone or in combination with ERBB inhibitors might abrogate the development of secondary drug resistance.

\section{Additional files}

\section{Additional file 1: Methods description.}

Additional file 2: Figure S1. (A) Schematic representation of the mechanism of wild-type or mutant EGFRvIll interactions for corresponding lanes in Figure 1A (numbered 2 to 13). Kinases were labeled with black (intact active centre) or red (kinase dead) and yellow (N-lobe mutant) or green (C-lobe mutant) circles. Intact or rescued kinase activity is represented by a phospho-tyrosine (P). (B) Wild-type (WT) or mutated EGFRvIII (KD-Kinase defective) were transfected into HEK293 cells either alone or in combinations as indicated. Untransfected cells (UT) were taken as a negative control (lane 1). Cell lysis was performed 36 hours after transfection followed by SDS-PAGE. Immunoblotting was performed with anti-p-EGFR (Y1068) and anti-EGFR antibodies.

Additional file 3: Figure S2. (A) Schematic representation of receptor interactions shown in Figure 1B. (B) Schematic representation of receptor interactions in the setting of both homo- and hetero-dimers. Numbers correspond to lanes in the Figure $2 \mathrm{~A}$.

\section{Competing interest}

The authors have no competing interests to declare.

\section{Authors' contributions}

RKK: Conceived the study, designed and performed experiments, analyzed data and wrote the manuscript. NvB: Conceived the study and analyzed data. JD: Conceived the study, analyzed data and wrote the manuscript. All authors read and approved the final manuscript.

\section{Acknowledgements}

Justus Duyster is supported by a grant from the José Carreras LeukämieStiftung. The authors thank Natalie Bartosch for technical support.

Received: 11 November 2012 Accepted: 27 May 2013

Published: 10 June 2013

\section{References}

1. Chu CT, Everiss KD, Wikstrand CJ, Batra SK, Kung HJ, Bigner DD: Receptor dimerization is not a factor in the signalling activity of a transforming variant epidermal growth factor receptor (EGFRvill). Biochem J 1997, 324(Pt 3):855-861.

2. Wikstrand CJ, Reist CJ, Archer GE, Zalutsky MR, Bigner DD: The class III variant of the epidermal growth factor receptor (EGFRvIII): characterization and utilization as an immunotherapeutic target. J Neurovirol 1998, 4(2):148-158.

3. Hwang $Y$, Chumbalkar V, Latha $K$, Bogler O: Forced dimerization increases the activity of DeltaEGFR/EGFRvIll and enhances its oncogenicity. Mol Cancer Res 2011, 9(9):1199-1208.

4. Gajadhar AS, Bogdanovic E, Munoz DM, Guha A: In situ analysis of mutant EGFRs prevalent in glioblastoma multiforme reveals aberrant dimerization, activation, and differential response to anti-EGFR targeted therapy. Mol Cancer Res 2012, 10(3):428-440.

5. Fernandes H, Cohen S, Bishayee S: Glycosylation-induced conformational modification positively regulates receptor-receptor association: a study with an aberrant epidermal growth factor receptor (EGFRvIll/DeltaEGFR) expressed in cancer cells. J Biol Chem 2001, 276(7):5375-5383.
6. Pines $G$, Huang PH, Zwang $Y$, White FM, Yarden Y: EGFRvIV: a previously uncharacterized oncogenic mutant reveals a kinase autoinhibitory mechanism. Oncogene 2010, 29(43):5850-5860.

7. Zhang X, Gureasko J, Shen K, Cole PA, Kuriyan J: An allosteric mechanism for activation of the kinase domain of epidermal growth factor receptor. Cell 2006, 125(6):1137-1149.

8. Kancha RK, von Bubnoff N, Peschel C, Duyster J: Functional analysis of epidermal growth factor receptor (EGFR) mutations and potential implications for EGFR targeted therapy. Clin Cancer Res 2009, 15(2):460-467.

9. Kancha RK, Peschel C, Duyster J: The epidermal growth factor receptorL861Q mutation increases kinase activity without leading to enhanced sensitivity toward epidermal growth factor receptor kinase inhibitors. J Thorac Oncol 2011, 6(2):387-392

10. Ymer SIG, Sameer A, Cvrljevic A, Cao DX, Donoghue JF, Epa VC, Scott AM, Adams TE, Johns TG: Glioma specific extracellular missense mutations in the first cysteine rich region of epidermal growth factor receptor (EGFR) initiate ligand independent activation. Cancers 2011, 3(2):2032-2049.

11. Jura N, Shan Y, Cao X, Shaw DE, Kuriyan J: Structural analysis of the catalytically inactive kinase domain of the human EGF receptor 3. Proc Natl Acad Sci USA 2009, 106(51):21608-21613.

12. Kancha RK, von Bubnoff N, Bartosch N, Peschel C, Engh RA, Duyster J: Differential sensitivity of ERBB2 kinase domain mutations towards lapatinib. PLoS One 2011, 6(10):e26760.

13. Wang Z, Longo PA, Tarrant MK, Kim K, Head S, Leahy DJ, Cole PA: Mechanistic insights into the activation of oncogenic forms of EGF receptor. Nat Struct Mol Biol 2011, 18(12):1388-1393.

14. Macdonald-Obermann JL, Piwnica-Worms D, Pike L: Mechanics of EGF receptor/ErbB2 kinase activation revealed by luciferase fragment complementation imaging. Proc Natl Acad Sci USA 2012, 109(1):137-142.

15. Kani K, Warren CM, Kaddis CS, Loo JA, Landgraf R: Oligomers of ERBB3 have two distinct interfaces that differ in their sensitivity to disruption by heregulin. J Biol Chem 2005, 280(9):8238-8247.

16. Xu W, Mimnaugh E, Rosser MF, Nicchitta C, Marcu M, Yarden Y, Neckers L: Sensitivity of mature Erbb2 to geldanamycin is conferred by its kinase domain and is mediated by the chaperone protein Hsp90. J Biol Chem 2001, 276(5):3702-3708.

17. Huang X, Gao L, Wang S, McManaman JL, Thor AD, Yang X, Esteva FJ, Liu B: Heterotrimerization of the growth factor receptors erbB2, erbB3, and insulin-like growth factor-i receptor in breast cancer cells resistant to herceptin. Cancer Res 2010, 70(3):1204-1214.

doi:10.1186/1478-811X-11-39

Cite this article as: Kancha et al:: Asymmetric kinase dimer formation is crucial for the activation of oncogenic EGFRvill but not for ERBB3 phosphorylation. Cell Communication and Signaling 2013 11:39.

\section{Submit your next manuscript to BioMed Central and take full advantage of:}

- Convenient online submission

- Thorough peer review

- No space constraints or color figure charges

- Immediate publication on acceptance

- Inclusion in PubMed, CAS, Scopus and Google Scholar

- Research which is freely available for redistribution 